Dhaka Univ. J. Biol. Sci. 29(1): 37-44, 2020 (January)

\title{
ADAPTATION OF THE SELF-COMPASSION SCALE FOR BANGLADESHI CULTURE
}

\author{
Sumaiya Habib and Jamiun NAHeR* \\ Department of Psychology, University of Dhaka, Dhaka-1000, Bangladesh
}

Key words: Self compassion, Reliability, Validity, Factor analysis

\begin{abstract}
Self-compassion is a raising construct in the field of positive psychology. Evidence supported that self-compassion is an important factor in increasing psychological well-being and reducing symptoms of anxiety, depression and rumination. In the present study, authors determined the psychometric properties of the Bangla version of Self-Compassion Scale (SCS). The study was carried out in a sample of 160 adults, ranging from 18 to 28 years old. Although the original scale has 26 items, in item-total correlation analysis it was found that 24 items in the questionnaire were significantly correlated with total items, the rest two items consumed negative/ow correlation (below 0.30). So, these two items were excluded from the Bangla version and 24 items were selected for the final version of the SCS. The internal consistency of the SCS was computed by Cronbach's Alpha which was 0.83 and the split-half coefficient of the scale was 0.84. Thus the results support the Bangla SCS is suitable for use in Bangladeshi culture.
\end{abstract}

\section{Introduction}

Self-compassion is theme of Buddhist psychology for centuries and it has become the focus of eastern and western psychologies(1). The term "Self-compassion" derives from the word "compassion", which refers to an understanding of another's pain and the desire to alleviate that pain. Directing the same compassionate attitude towards oneself involves self-compassion. Self-compassion is defined as treating oneself kindly in moments of failure and acknowledging negative feelings with openness and acceptance $^{(1)}$. It works as buffer against negative coping such as self-criticism and acts as a protective factor against mental illness. It involves being kind, compassionate and understood toward oneself when one is emotionally distressed or when there is a feeling of incompetence or failure ${ }^{(2)}$. There are three interrelated components of selfcompassion. These components might be of great help in times of suffering, if it is well trained. The three main elements include: (i) Self-kindness - being supportive , kind and caring toward oneself rather than being harshly self-critical when individuals having a

\footnotetext{
*Author for correspondence: <naherjamiun31@gmail.com>.
} 
hard time, (ii) common humanity - realizing the pain and suffering as a part of larger human experience rather than isolating oneself from others, (iii) mindfulness - recognition of one's negative emotions without being judgmental and accepting present moment ${ }^{(1,2)}$.

Researchers have distinguished between self-compassion and self-esteem; the selfesteem involves personal evaluation, whereas self-compassion is the acknowledgment of shared human character. Moreover, self-compassion is not the same as selfindulgence; self-compassionate individuals acknowledge and accept their mistakes without suppressing them and that is different from self-indulgence ${ }^{(3)}$. Self- compassion is also different from self-pity. In this regard, self-pitying individuals become selfabsorbed in their personal problems and disregard others, whereas even when selfcompassionate individuals facing personal problems, they tend to be available for others. Recent research has exhibited a relationship between self-compassion and psychological health, it has positive effect on happiness, optimism, conscientiousness, wisdom, curiosity, agreeableness, extraversion and decreased anxiety, depression, rumination ${ }^{(4)}$. A study showed that resilient adolescents and young adults practiced selfcompassion regularly and also found that family functioning, maternal support and attachment styles were predictors of self-compassion ${ }^{(5)}$. A body of research demonstrates that practicing self-compassion has facilitated a number of important health behaviors. For instance, self-compassion is associated with exercise behavior ${ }^{(6)}$, healthy eating behaviors ${ }^{(7)}$, medical adherence ${ }^{(8)}$, dietary adherence ${ }^{(9)}$ and indices of general healthpromoting behaviors(10). A nascent body of evidence demonstrated that selfcompassionate individuals experiencing greater psychological soundness than those who lack self-compassion. Evidence showed that self-compassion is linked to greater life satisfaction, personal growth, social connectedness, emotional resilience, autonomy, optimism and less anxiety, self-criticism, depression, rumination, neuroticism, thought suppression and perfectionism ${ }^{(1)}$. Self-compassion is regarded as beneficial in helping parents cope with the challenges that emerge when caring a disabled child(11).

To adapt the SCS authors can provide compassion exercise and meditation that lead to improve mood, more altruistic behavior, reduced stress, less anger, and reduced symptoms of post-traumatic stress disorder. Moreover, practicing self-compassion allows us to cultivate empathy, kindness and understanding toward ourselves and to improve our overall quality of life and vitality.

Unfortunately, there is no valid scientific tool to measure self-compassion in Bangladeshi culture. So, considering the significance of the tool, the aim of the present study is to adapt SCS into Bangla language and to validate it in our culture through psychometric testing. 


\section{Materials and Methods}

The sample consisted of 160 adult respondents (63 males, 97 females), aging from 19 to 28 years old were taken following purposive and convenience sampling technique. All of the respondents were students, studying in the University of Dhaka.

For the data collection, following materials were used: (a) Demographic and personal information questionnaire, (b) Bangla version of self-compassion Scale.

Self-compassion is assessed using the self-compassion scale which had been developed by Kristin Neff in order to measure compassion towards oneself ${ }^{(1)}$. The original scale has 26 items which has six subscales include the Self-Kindness subscale (5 items), the Self-Judgment subscale (5 items), the common humanity subscale (4 items), the isolation subscale ( 4 items), the Mindfulness subscale (4 items) and the Overidentification subscale (4 items). Responses are given on a 5-point scale from "1-Almost Never" to "5-Almost Always." The original SCS has high internal reliability $(\alpha=0.90)$ and test-retest consistency $(0.93)^{(1)}$. A total SCS is computed by reversing the negative subscale items and then adding all the subscale subsequently which may range from 24 to 120 , with higher scores indicating greater self-compassion.

The adaptation of SCS involved several multistage procedures following the cross cultural standard guideline regarding adaptation and validation of psychometric instrument ${ }^{(12)}$. The items of SCS were translated into considering the meaning, clarity and simplicity of the items. Then blind back translation was done by two faculty members of Department of Psychology, University of Dhaka. The translated version and the back translated version were reviewed by two experts, proficient in both Bengali and English language. The synthesis version was then administered to 25 students to examine the strength of items concerning their meaning and difficulty. The scale was finalized after the pilot study. The final translation of SCS was administered to 160 students from University of Dhaka.

\section{Results and Discussion}

Item analysis of a Likert-type scale is done by calculating the correlation between individual item's score with the total scale score. During pilot study, all 26 items were thoroughly analyzed and corrected item total correlation coefficients were determined. From those corrected item-total correlation value, SCS_ 8 (-0.398) and SCS_21 (-0.267) were detected as negative or low correlation score $(\mathrm{r}<0.03)$ with total score indicates that the items were defective in measuring what the whole SCS questionnaire was supposed to measure. These two items required further review to accept it in the Bangla version of SCS. The rest 24 items were found to have significant (at 0.05) correlation coefficients shown in Table 1. So it was concluded that these 24 items had satisfactory level of internal consistency and selected for the final Bangla version of Self-compassion Scale. 
The Cronbach's Alpha was found 0.83 for 160 cases and the odd-even Split-half reliability coefficient was found 0.84 after using the Spearman-Brown correlation formula (Table 2). These reliability coefficients proved the Bangla version of the Self-Compassion Scale (SCS) to be a reliable scale.

Table 1. Item-total statistics and Cronbach's alpha if the item is deleted.

\begin{tabular}{ccc}
\hline Items & $\begin{array}{c}\text { Corrected item-total } \\
\text { correlation }\end{array}$ & $\begin{array}{c}\text { Cronbach's alpha if item } \\
\text { deleted }\end{array}$ \\
\hline SCS_1 & 0.133 & 0.836 \\
SCS_2 & 0.314 & 0.829 \\
SCS_3 & 0.358 & 0.827 \\
SCS_4 & 0.593 & 0.816 \\
SCS_5 & 0.406 & 0.825 \\
SCS_6 & 0.447 & 0.823 \\
SCS_7 & 0.439 & 0.824 \\
SCS_8 & 0.398 & 0.826 \\
SCS_9 & 0.348 & 0.828 \\
SCS_10 & 0.496 & 0.822 \\
SCS_11 & 0.392 & 0.830 \\
SCS_12 & 0.496 & 0.822 \\
SCS_13 & 0.439 & 0.824 \\
SCS_14 & 0.521 & 0.821 \\
SCS_15 & 0.317 & 0.829 \\
SCS_16 & 0.311 & 0.829 \\
SCS_17 & 0.432 & 0.824 \\
SCS_18 & 0.297 & 0.830 \\
SCS_19 & 0.154 & 0.835 \\
SCS_20 & 0.294 & 0.829 \\
SCS_21 & 0.191 & 0.833 \\
SCS_22 & 0.476 & 0.822 \\
SCS_23 & 0.444 & 0.824 \\
SCS_24 & 0.496 & 0.822 \\
\hline
\end{tabular}

Table 2. Reliability coefficient of the Bangla version of SCS.

\begin{tabular}{|c|c|c|}
\hline Scale & Cronbach's alpha reliability coefficient $(n=160)$ & Split-half reliability \\
\hline $\begin{array}{l}\text { Self-Compassion } \\
\text { Scale }\end{array}$ & $0.83^{*}$ & $0.84^{*}$ \\
\hline
\end{tabular}


Face and content validity of the scale were assured by checking items during construction of the test. Principle component analysis was used to determine the construct validity of the scale. The Scree plot of the principle component analysis (Fig. 1) showed that scale has Eigen values greater than 1. The Eigen values of 24 items of the SCS are shown in Table 3.

Table 3. Total variance explained.

\begin{tabular}{cccc}
\hline Component & \multicolumn{3}{c}{ Initial Eigen values } \\
\cline { 2 - 4 } & Total & \% of variance & Cumulative \% \\
\hline 1 & 5.343 & 22.262 & 22.262 \\
2 & 3.267 & 13.613 & 35.875 \\
3 & 1.389 & 5.786 & 41.661 \\
4 & 1.211 & 5.046 & 46.707 \\
5 & 1.153 & 4.803 & 51.510 \\
6 & 1.102 & 4.590 & 56.100 \\
7 & 1.013 & 4.222 & 60.322 \\
8 & 0.982 & 4.091 & 64.412 \\
9 & 0.842 & 3.508 & 67.920 \\
10 & 0.828 & 3.452 & 71.372 \\
11 & 0.761 & 3.170 & 74.541 \\
12 & 0.724 & 3.016 & 77.557 \\
13 & 0.678 & 2.824 & 80.381 \\
14 & 0.568 & 2.366 & 82.748 \\
15 & 0.551 & 2.297 & 85.045 \\
16 & 0.526 & 2.190 & 87.234 \\
17 & 0.493 & 2.055 & 89.290 \\
18 & 0.467 & 1.946 & 91.236 \\
19 & 0.424 & 1.766 & 93.002 \\
20 & 0.406 & 1.690 & 94.692 \\
21 & 0.386 & 1.610 & 96.302 \\
22 & 0.328 & 1.365 & 97.667 \\
23 & 0.286 & 1.190 & 98.857 \\
24 & 0.274 & 1.143 & 100.000 \\
\hline
\end{tabular}

Extraction method: Principal component analysis.

The component matrix analysis found 7 factors. In the original scale there were 6 subscales. May be there are a bit more variability in the data collected for adaptation process. Data of the factorial validity are shown in Table 4. 
Table 4. Component matrix.

\begin{tabular}{|c|c|c|c|c|c|c|c|}
\hline & \multicolumn{7}{|c|}{ Component } \\
\hline & 1 & 2 & 3 & 4 & 5 & 6 & 7 \\
\hline SCS1 & 0.137 & 0.279 & 0.465 & -0.080 & 0.147 & 0.524 & 0.422 \\
\hline SCS2 & 0.345 & 0.590 & 0.254 & -0.033 & -0.013 & -0.211 & 0.020 \\
\hline SCS3 & 0.471 & -0.549 & -0.012 & 0.114 & -0.006 & -0.196 & 0.302 \\
\hline SCS4 & 0.642 & 0.382 & -0.001 & 0.121 & -0.075 & 0.027 & 0.135 \\
\hline SCS5 & 0.504 & -0.232 & 0.227 & 0.027 & -0.368 & -0.247 & 0.029 \\
\hline SCS6 & 0.498 & 0.365 & 0.272 & 0.098 & -0.035 & 0.041 & -0.406 \\
\hline SCS7 & 0.548 & -0.236 & -0.103 & -0.236 & -0.194 & -0.042 & 0.169 \\
\hline SCS8 & 0.509 & -0.249 & -0.138 & -0.403 & 0.246 & -0.170 & 0.040 \\
\hline SCS9 & 0.441 & -0.378 & -0.084 & 0.098 & 0.502 & 0.060 & 0.276 \\
\hline SCS10 & 0.566 & 0.177 & 0.336 & -0.388 & 0.185 & 0.051 & -0.171 \\
\hline SCS11 & 0.440 & 0.437 & -0.120 & 0.345 & 0.133 & 0.166 & 0.046 \\
\hline SCS12 & 0.598 & -0.159 & -0.273 & 0.064 & 0.038 & 0.013 & -0.322 \\
\hline SCS13 & 0.543 & -0.468 & -0.060 & 0.097 & 0.105 & 0.240 & 0.071 \\
\hline SCS14 & 0.581 & 0.380 & 0.174 & -0.166 & -0.057 & -0.104 & 0.032 \\
\hline SCS15 & 0.414 & -0.398 & 0.259 & 0.117 & -0.145 & -0.113 & -0.107 \\
\hline SCS16 & 0.349 & 0.433 & -0.425 & 0.187 & 0.148 & 0.122 & 0.073 \\
\hline SCS17 & 0.554 & -0.304 & -0.064 & -0.074 & -0.172 & -0.191 & 0.115 \\
\hline SCS18 & 0.345 & 0.497 & -0.227 & -0.086 & 0.239 & -0.229 & -0.151 \\
\hline SCS19 & 0.225 & -0.251 & -0.054 & -0.256 & -0.405 & 0.610 & -0.152 \\
\hline SCS20 & 0.385 & -0.376 & -0.190 & 0.333 & -0.186 & 0.129 & -0.087 \\
\hline SCS21 & 0.257 & -0.362 & 0.528 & 0.479 & 0.220 & -0.073 & -0.116 \\
\hline SCS22 & 0.540 & 0.360 & -0.104 & -0.122 & -0.232 & -0.084 & 0.319 \\
\hline SCS23 & 0.470 & 0.488 & -0.134 & 0.319 & -0.250 & 0.084 & -0.003 \\
\hline SCS24 & 0.600 & -0.257 & -0.084 & -0.212 & 0.264 & 0.183 & -0.326 \\
\hline
\end{tabular}

Extraction method: Principal component analysis.

a. 7 components extracted.

In order to analysis the appropriateness of data (adequacy), authors also conducted KMO and Bartlett's test. In Table 5, it was noticed that the KMO value (0.815) of this present study was greater than the desired level $(\mathrm{KMO}>0.50)$. So, it could be concluded that authors had good sampling by which the variables could be factored. Bartlett's test 
also showed that our correlation matrix is not an identity matrix (as Sig. < 0.05) which is significant for factor analysis meets the criteria necessary to proceed.

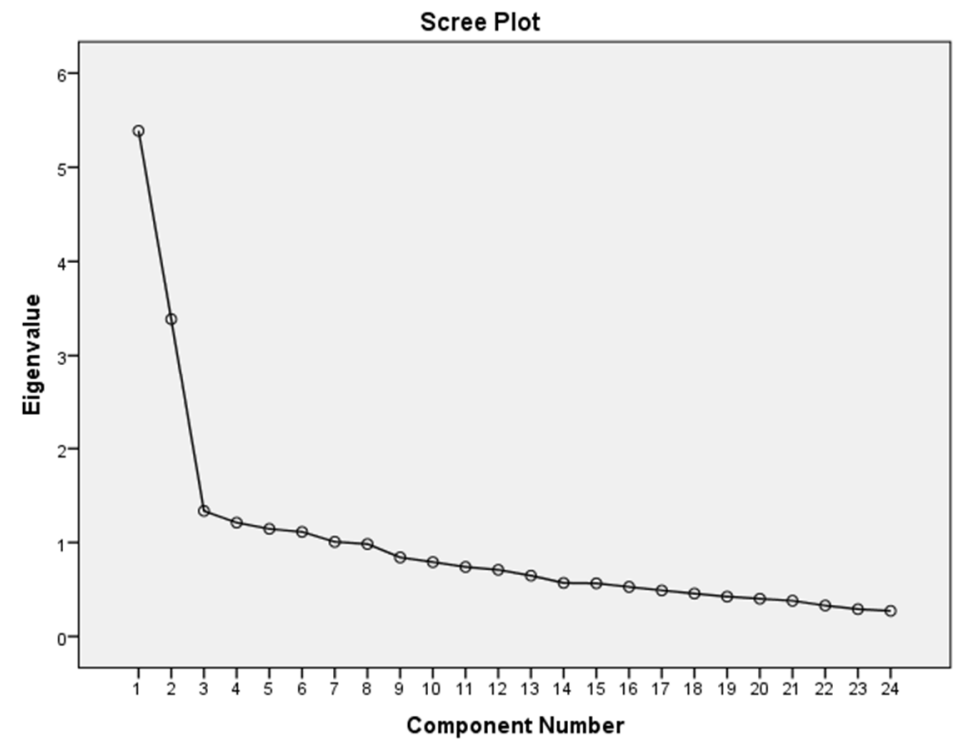

Fig. 1. Scree plot of Eigen values of scale components.

Table 5. KMO and Bartlett's test.

\begin{tabular}{lll}
\hline $\begin{array}{l}\text { Kaiser-Meyer-Olkin measure of sampling } \\
\text { adequacy }\end{array}$ & 0.815 \\
\hline $\begin{array}{l}\text { Bartlett's test of } \\
\text { sphericity }\end{array}$ & Approx. Chi-square & 1131.857 \\
& df & 276 \\
& Sig. & 0.000 \\
\hline
\end{tabular}

Thus the adapted Bangla scale proved to be a reliable and valid measure of selfcompassion. So the mental health professionals and the counselors can use this scale and can also provide self-compassion mediation and exercise to their clients. However, the study has a number of recommendations. The scale needs to be further examined in larger and more diverse populations. Additionally the scale's sensitivity and discriminant validity should be undertaken in future to provide sufficient proof of its appropriateness in Bangladeshi culture and population.

\section{References}

1. Neff KD 2003. Development and validation of a scale to measure self- compassion. Self and Identity 2: 223-250. 
2. Neff KD 2003b. Self-compassion : An alternative conceptualization of a healthy attitude toward oneself. Self and Identity 2: 85-102. doi: 10.1080/15298860390129863.

3. Barnard LK. and JF Curry 2011. Self-compassion: Conceptualizations, correlates, and interventions. Review of General Psychology 15: 289-303.

4. Neff KD 2009a. The role of self-compassion in development: A healthier way to relate to oneself. Human Development 52 : 211-214. doi: 10.1159 000215071.

5. Neff KD and P McGehee 2010. Self-compassion and psychological resilience among adolescents and young adults. Self and Identity 9: 225-240.

6. Magnus CMR, KC Kowalski and T-LF McHugh 2010. The role of self-compassion in women's self-determined motives to exercise and exercise-related outcomes. Self and Identity 9(4): 363-382.

7. Adams CE and MR Leary 2007. Promoting self-compassionate attitudes toward eating among restrictive and guilty eaters. Journal of Social and Clinical Psychology 26(10): 1120-1144.

8. Brion J, M Leary and A Drabkin 2014. Self-compassion and reactions to serious illness: The case of HIV. Journal of Health Psychology 19(2): 218-229.

9. Dowd AJ and ME Jung 2017. Self-compassion directly and indirectly predicts dietary adherence and quality of life among adults with celiac disease. Appetite 113: 293-300.

10. Sirois FM, R Kitner and JK Hirsch 2015a. Self-compassion, affect, and health behaviors. Health Psychology 34(6): 661-669.

11. Robinson S , RP Hastings, JA Weiss, J Pagavathsing and Y Lunsky 2017. Self-intellectual and developmental disabilities. Journal of Applied Research in Intellectual Disabilities 31(3): 454-458.

12. Beaton DE, C Bombardier, F Guillemin and M Bosi-Ferraz 2000. Guidelines for the process of cross-cultural adaptation of self-report measure. Spine 25: 3186-3191.

(Manuscript received on 11 April, 2019; revised on 6 May, 2019) 